\title{
Psicoterapia del oprimido
}

Daniel Rzondzinski

Martin Luther University College (Canada) 



\title{
Psicoterapia del oprimido
}

\section{Psychotherapy of the oppressed}

\author{
Daniel Rzondzinski \\ Martin Luther University College (Canada) \\ danielrzo@hotmail.com
}

Fecha de recepción: 28 de septiembre de 2017

Fecha de aceptación: 27 de diciembre de 2019

\begin{abstract}
Resumen
Este artículo trata de analizar cómo se puede enseñar y aprender el concepto de relación terapéutica siendo éste término, el contenido central de la asignatura Pre-Práctica, materia previa y obligatoria para los estudiantes que deben realizar su práctica de residencia como psicoterapeutas en la carrera de Maestría en Espiritualidad y Psicoterapia de la Universidad Wilfrid Laurier de la provincia de Ontario, Canadá. El concepto de relación terapéutica es abordado desde las perspectivas teóricas del pensamiento de Piaget, Freire y Morin, siendo dicha relación un elemento esencial que permite la implementación del proceso de psicoterapia y de los cambios que el paciente necesita realizar para mejorar su salud mental. A su vez, se analizan los conceptos de transferencia, contratransferencia y alianza terapéutica desde la perspectiva dinámica y cognitiva. Este artículo está sustentado teórica y conceptualmente en los principios de la transdiciplinariedad y del pensamiento complejo.
\end{abstract}

Palabras clave: Educación universitaria; Relación terapéutica; Transferencia; Alianza terapéutica; Pensamiento complejo

\begin{abstract}
This article describes how to teach and how to learn about the nature of the therapeutic relationship. This concept is the main content of the Pre-Practicum course which is a cumpulsory prerequisite for students before they begin their practicum as psychotherapists in the Master in Spirtuality and Psychotherapy degree program of Wilfrid Laurier University in the province of Ontario, Canada.
\end{abstract}


Psicoterapia del oprimido...- D. Rzondzinski

The concept of therapeutic relationship is analyzed from the perspective of Piaget, Freire and Morin. This article is focused on the importance and the needs of reaching a good therapeutic relationship in order to implement the psychotherapy process and the changes that the client needs to achieve to improve his mental health. This work analyzes the concepts of transference, countertransference and therapeutic alliance from dynamic and cognitive perspectives. This article is supported by the principles of transdisciplinarity and complex thinking.

Keywords: University education; Therapeutic relationship; Transference; Therapeutic alliance; Omplex thinking

\section{PRESENTACIÓN}

El artículo que se presenta al lector es parte de una propuesta doctoral en curso dirigida por el Doctor Israel Sandre Osorio de la Multiversidad Mundo Real Edgar Morin de México. Este artículo trata de analizar cómo se puede enseñar y aprender el concepto de relación terapéutica, siendo éste término, el contenido central de la asignatura Pre-Práctica, asignatura previa y obligatoria a la práctica de residencia como psicoterapeuta que se dicta en la carrera de Maestría en Espiritualidad y Psicoterapia de la Universidad Wilfrid Laurier de la provincia de Ontario, Canadá. Este artículo pretende analizar la naturaleza de la relación terapéutica (relación entre el psicoterapeuta y el paciente) teniendo en cuenta los conceptos de transferencia, contratransferencia, alianza terapéutica y poder.

El resultado del análisis pretende concientizar al estudiante acerca de la presencia del uso del poder en toda relación terapéutica. Esto significa que toda relación terapéutica puede constituirse en un instrumento de liberación para el paciente o en un instrumento de opresión y dominación. Si el estudiante como futuro psicoterapeuta comprende el uso negativo del poder, es decir, poder usado para beneficio de la enfermedad y no para beneficio de la salud mental del paciente; podrá mejorar la calidad de sus intervenciones clínicas (esto significa poder efectivamente ayudar al paciente promoviendo su autoestima, autonomía y toma de decisiones por si mismo, reduciendo la influencia negativa de su enfermedad) ya sea desde una perspectiva dinámica, cognitiva o cualquier otra. Este artículo se sustenta teórica y conceptualmente en el pensamiento de Piaget, Freire y Morin con el objetivo de comprender, de enseñar y aprender el concepto de relación terapéutica, teniendo en cuenta la construcción del conocimiento formulada por Piaget, la crítica política de Freire acerca de la educación tradicional y el pensamiento complejo, integrador de Morin sobre saberes disciplinarios separados y a veces, en oposición que debieran estar unidos. 


\section{JUSTIFICACIÓN}

La relación terapéutica, la relación entre el terapeuta y el paciente es de suma importancia en el proceso de psicoterapia y en los cambios que el paciente puedarealizar con el fin de mejorar su salud mental. La calidad de la relación terapéutica, es decir, la calidad de la relación entre el terapeuta y el paciente puede afectar el tratamiento y su resultado. Si la calidad de la relación terapéutica es positiva, el tratamiento y su resultado también lo serán. Durante cualquier tratamiento se espera que el paciente pueda mejorar su autoestima, es decir, desarrollar una percepción positiva de sí mismo, pueda desarrollar la capacidad de tomar decisiones adecuadas, o sea, que lo beneficien y reducir al máximo la influencia negativa de la enfermedad. Por ello, la relación terapéutica es el contenido central del curso Pre-Práctica que los estudiantes deben aprender antes de iniciar su práctica de residencia como psicoterapeutas.

Se puede afirmar tal como lo indican Corbella y Botella (2009) qué si la relación terapéutica no es buena, si el paciente rechaza al terapeuta o el terapeuta rechaza al paciente consciente o inconscientemente, el paciente no podrá efectuar los cambios necesarios para enfrentar la psicopatología que lo aqueja e incluso puede potencialmente empeorar.

Si el paciente rechaza al terapeuta o el terapeuta rechaza al paciente, la relación terapéutica no puede construirse, consecuentemente el tratamiento no puede efectuarse. Si el terapeuta acepta al paciente y el paciente acepta al terapeuta, depertándose un afecto mutuo, la relación terapéutica se construye y el tratamiento comienza. Algunas veces, durante el tratamiento, la relación terapéutica se deteriora, generalmente por alguna intervención del terapeuta que no ha sido acertada. En algunos casos, el paciente puede pensar que su terapeuta no es lo suficientemente capaz para ayudarlo a resolver su problema o difiere con algunas ideas del terapeuta al punto de rechazarlo. En algunas situaciones una idea errónea del terapeuta, comunicada en una situación no adecuada al paciente (en psicoanálisis se denomina a esta intervención: interpretación fuera de contexto) puede ser internalizada en la mente del paciente empeorando la salud mental del paciente. Por ello, se puede deducir que el logro de una buena relación terapéutica al inicio y durante el tratamiento es el componente más importante de carácter curativo ${ }^{1}$ en el proceso de psicoterapia independientemente del tratamiento empleado con el paciente sea cognitivo, psicoanalítico o cualquier otro. A su vez, se puede afirmar que la calidad

1 En este artículo se considera el concepto de “cura” formulado por Lacan. En su Diccionario Introductorio de Psicoanálisis Lacaniano, Dylan Evans (2015), describe el concepto de cura en un sentido completamamente distinto al seguido por la medicina. La meta de la cura para Lacan no es sanar o curar en el sentido de producir una psique perfectamente sana. Las estructuras clínicas (neurosis, psicosis y perversión) son consideradas esencialmente incurables y el tratamiento psicoanalítico se propone únicamente llevar al analizante (paciente) a expresar su verdad subjetiva. 
de la relación terapéutica alcanzada entre el paciente y su terapeuta constituye un factor determinante en relación a los resultados a obtenerse del tratamiento. Debido a esto último, todos los modelos de tratamiento ponen enorme énfasis en el desarrollo de una buena relación terapéutica entre el terapeuta y su paciente.

Este artículo se propone explicar cómo el educador y los educandos construyen el concepto de relación terapéutica siguiendo el pensamiento de Piaget. Esto es partiendo de lo concreto a lo abstracto; partiendo de la vida cotidiana del alumno y docente, de su contexto social, de su participación en diversas relaciones sociales humanas y mediante un proceso gradual de abstracción, formulándose el concepto de relación terapéutica y estableciéndose sus similitudes y diferencias; es decir, su naturaleza, en contraposición con el conjunto de las relaciones humanas. A su vez, se analiza el uso del poder por parte del psicoterapeuta con el fin de liberar al paciente de la opresión ejercida por la enfermedad mental. Para ello, se toma en cuenta el pensamiento de Freire que centra su análisis en el uso del poder dentro del sistema educativo tradicional basado en la opresión y dominación. Este artículo afirma que existe un paralelismo entre el uso del poder opresivo en la relación terapéutica y el uso del poder opresivo en la relación pedagógica formulada por Freire.

Finalmente, se describe la relación terapéutica como un concepto transdisciplinario (En este trabajo serán consideradas como disciplinas separadas, porque en su uso lo son, las dos teorías o enfoques fundamentales de la psicoterapia actual: La teoría psicoanalítica o dinámica y la teoría cognitiva) siendo producto de los saberes provenientes del psicoanálisis (saber enfocado en el aspecto inconsciente de la relación terapéutica) y de la psicología cognitiva (saber enfocado en el aspecto consciente de la relación terapéutica) siguiéndose los principios del pensamiento complejo desarrollado por Morin (1999), la relación terapéutica está abordada por la psicoterapia actual de manera fragmentada mediante el uso del principio de simplificación. Para los psicoterapeutas psicoanalíticos la relación terapéutica es sólo inconsciente, y para los psicoterapeutas cognitivos la relación terapéutica es sólo consciente. Mediante el uso del pensamiento complejo desarrollado por Morín, se pretende articular, unir lo que está fragmentado, lo que en realidad debiera estar unido. La relación terapéutica no es sólo consciente. La relación terapéutica no es sólo inconsciente. En este trabajo se afirma la tesis de que la relación terapéutica es consciente e inconsciente a la vez, de la misma manera que la teoría del caos en la física afirma que el orden y el desorden son parte del mismo nivel de realidad.

En concordancia con lo arriba expuesto, se analiza los aportes y limitaciones del enfoque dinámico y del enfoque cognitivo en el desarrollo del concepto de relación terapéutica. Este análisis permite comprender que el enfoque dinámico o el enfoque cognitivo, por si mismos, no logran explicar completamente la naturaleza de la relación terapéutica y por lo tanto, al no comprendérsela en profundidad, muchas intervenciones clínicas, incluso de experimentados psicoterapeutas, seguidores de uno u otro enfoque, no suelen ser exitosas. 


\section{LA RELACIÓN TERAPÉUTICA}

La primera pregunta que requiere ser contestada es cómo el educador construye con sus educandos el concepto de relación terapéutica pudiendo establecer la diferencia entre los conceptos de transferencia, contratransferencia, alianza terapéutica y encuadre. Para ello, seguiremos a Badilla Saxe (2008) y su reflexión acerca del pensamiento de Piaget.

Piaget nos muestra cómo la forma de aprender, es comenzar interactuando con lo que nos es cercano, reconocible, significativo e ir evolucionando hacia conceptos más complejos. Dice que el aprendizaje se construye partiendo de esquemas concretos que van evolucionado hacia estructuras más abstractas. Y si bien es común que nuestro discurso educativo está teñido de esta propuesta piagetiana (de lo concreto a lo abstracto, repetimos con frecuencia) hacemos todo lo contrario. Los planes de estudio inician con lo más abstracto (los fundamentos) y se reserva para el final lo concreto (la práctica). El estudiantado va pasando los cursos, a pesar de esta contradicción, pero debemos confesar que les ponemos dificultades innecesarias (Badilla Saxe, 2008, p.1)

Siguiendo las ideas formuladas por Badilla Saxe en el curso de Pre-Práctica no se hace teoría primero y práctica después. La teoría y la práctica se efectúan simultáneamente. El educador y sus educandos comienzan reflexionando acerca de la relación terapéutica al interactuar con lo que les es más cercano, reconocible, significativo para luego evolucionar hacia conceptos más complejos. El pasaje de lo concreto a lo abstracto se logra mediante el pasaje de las relaciones interpersonales (lo concreto) a la relación terapéutica (lo abstracto). Aquí se entiende a las relaciones interpersonales como aquellas que se dan entre las personas en su vida cotidiana. Entre ellas mayormente encontramos a las relaciones profesionales, las relaciones de amistad, las relaciones amorosas y las relaciones sexuales. Educador - educandos siguiendo un enfoque piagetiano reflexionan y debaten acerca de sus relaciones interpersonales con el fin de posteriormente diferenciar las relaciones interpersonales en general, de la relación terapéutica en particular.

Inicialmente se puede afirmar que la relación terapéutica a diferencia del resto de las relaciones humanas, se caracteriza por presentar siete aspectos fundamentales que se enuncian y se explican a continuación:

1. La relación terapéutica es una relación intersubjetiva.

2. La relación terapéutica es una relación profesional pero diferente a otras relaciones profesionales.

3. La relación terapéutica no es una relación de amistad.

4. La relación terapéutica no es una relación amorosa.

5. La relación terapéutica no incluye relaciones sexuales. 
Psicoterapia del oprimido...- D. Rzondzinski

6. La relación terapéutica es siempre una relación de poder.

7. La relación terapéutica siempre incluye fenómenos transferenciales, contratransferenciales, de encuadre y de alianza terapéutica.

8. La relación terapéutica es un sistema adaptativo complejo.

\subsection{La relación terapéutica es una relación intersubjetiva}

La relación terapéutica es una relación intersubjetiva. Esta afirmación se sostiene a partir del concepto de interseccionalidad que fue formulado por Wiliams Crenshaw en 1989 donde se afirma la existencia de identidades sociales ocultas en intersección, con sus correspondientes sistemas de opresión; dado que la relación terapéutica (terapeuta - paciente) como la relación pedagógica (educador-educando) presentan la interacción de por lo menos dos subjetividades, es decir, la subjetividad del terapeuta y la subjetividad del paciente como así también, la subjetividad del educador y la subjetividad del educando y que más allá de sus posiciones psicológicas subjetivas definas por Lacan'; existen entre ellos diferencias que pueden formularse en relación a su ubicación social. Esto es la pertenencia a una determinada clase social, raza, religión, nivel de educación, género, orientación sexual, afiliación política, cultura de pertenencia, etc. La interacción de subjetividades a partir de los factores mencionados, es decir, la intersubjetividad, puede facilitar el funcionamiento de la relación terapéutica u obstaculizarla. Situación similar ocurre en el seno de la relación pedagógica entre educador y educando. Por ello, la potencial discriminación como forma de opresión basada en estos factores por parte de las subjetividades intervinientes en el proceso psicoterapéutico como en el proceso educativo debe ser considerada. Especialmente cuando dicha discriminación proviene de quién posee una posición de poder debido al rol que ocupa, ya sea de terapeuta o de educador.

En este apartado educador y educandos debaten y deconstruyen el concepto de interseccionalidad con la finalidad de explorar su posición subjetiva respecto al mismo debido a que están inmersos en una doble relación. Esto es la relación pedagógica (educador - educando) que mantienen en la clase y la relación terapéutica (terapeuta - paciente) que deberán establecer en clase como trabajo práctico y fuera de clase en la práctica de residencia con sus pacientes.

\subsection{La relación terapéutica es una relación profesional}

La relación terapéutica es una relación profesional pero diferente a otras relaciones profesionales. La relación terapéutica es una relación de carácter artificial

2 Lacan describe tres posiciones subjetivas: la neurosis, la perversión y la psicosis. Estas posiciones subjetivas se definen en relación a cómo cada sujeto humano procesa su pasaje por el complejo de Edipo y el complejo de castración determinado su forma de goce. 
que tiene dos componentes: El terapeuta y su paciente. La relación terapéutica tiene por objeto el mejoramiento de la salud mental del paciente. Esto se logra mediante un trabajo psicológico conjunto entre terapeuta y paciente con el fin de que el paciente pueda liberarse de su enfermedad.

La relación terapéutica es similar a la relación pedagógica. Esto es así porque el proceso psicoterapéutico también, es un proceso educativo, que debe basarse en el uso del diálogo. Este diálogo entre terapeuta y paciente tiene por objetivo esclarecer la opresión del paciente por la enfermedad mental y/o su contexto social con la finalidad de que pueda liberarse, de igual manera que el educador y su educando deben trabajar conjuntamente para liberarse de la opresión que se hace presente en su contexto social y durante el proceso de aprendizaje. Estos procesos llevan a las personas involucradas a una verdadera transformación.

La existencia, en tanto humana, no puede ser muda, silenciosa, ni tampoco nutrirse de falsas palabras sino de palabras verdaderas con las cuales los hombres transforman el mundo. Existir humanamente, es "pronunciar" el mundo, es transformarlo. El mundo pronunciado, a su vez, retoma problematizado a los sujetos pronunciantes, exigiendo de ellos un nuevo pronunciamiento. (Freire, 1985. p. 100).

La transformación mencionada se logra a través de un proceso que Freire denomina concietización. Es decir, lograr comprender la naturaleza de la opresión para poder combatirla. No obstante, hay terapeutas que se colocan en el lugar del saber del paciente, partiendo de un concepto que Freire denominó educación bancaria. Esto es considerar al educador - terapeuta como poseedor del saber acerca del educando - paciente teniendo el derecho a depositar su conocimiento en una cuenta bancaria vacía que es la mente del educando - paciente. De esta forma el diálogo está ausente. Freire se propone la eliminación de la dicotomía educador educando, también siguiendo esa línea de pensamiento se podría llegar a considerar la eliminación de la dicotomía terapeuta - paciente.

En vez de comunicarse, el educador hace comunicados y depósitos que los educandos, meras incidencias, reciben pacientemente, memorizan y repiten. Tal es la concepción "bancaria" de la educación, en que el único margen de acción que se ofrece a los educandos es el de recibir los depósitos, guardarlos y archivarlos. Margen que sólo les permite ser coleccionistas o fichadores de cosas que archivan. (Freire, 1985, p. 72).

Esta actitud lleva a que el educando y al paciente a no poder pensar por sí mismos reduciendo su capacidad creativa para aprender un nuevo conocimiento que los libere de la opresión de la que sufren.

Educadores y educandos se archivan en la medida en que, en esta visión distorsionada de la educación (o la psicoterapia agrego), no existe creatividad alguna, no existe transformación, ni saber. Sólo existe saber en la invención, en la reinvención, en la búsqueda inquieta, impaciente, permanente, que los hombres realizan en el mundo, 
Psicoterapia del oprimido...- D. Rzondzinski

con el mundo y con los otros. Búsqueda que es también esperanzada. (Freire, 1985, pp. 72-73).

El educando no es una tabla rasa al igual que el paciente. El educando no necesita ser llenado por los conocimientos del educador tradicional. El educando sabe acerca de su opresión y el paciente es mejor experto en su enfermedad que el más brillante y experto psicoterapeuta. Por lo tanto, se debe partir de los conocimientos previos, de las condiciones sociales de opresión, de las condiciones opresivas de la enfermedad, antes de empezar un proceso de aprendizaje o de establecerse un diagnóstico y tratamiento.

En este apartado el educador y sus educandos deconstruyen el concepto de profesional (terapeuta experto - educador tradicional). Reflexionan acerca de la falta de salud mental como forma de opresión y la diferencian de otras opresiones de carácter social. Exploran la naturaleza artificial y educativa del proceso terapéutico. Reflexionan acerca de la importancia del dialogo como herramienta anti-opresiva y la potencial transformación de los intervinientes en la relación terapéutica como así también en la relación pedagógica. Deconstruyen el concepto de concientización y de educación bancaria y analizan como este tipo de educación produce opresión social limitando la capacidad pensante y creativa del educando y del paciente. Se cuestionan el rol del terapeuta experto y el rol del educador tradicional.

\subsection{La relación terapéutica no es una relación de amistad}

La relación terapéutica no es una relación de amistad porque la amistad incluye una conexión de carácter afectivo y una historia personal compartida. Si el terapeuta está envuelto histórica y afectivamente con el paciente, dicho vínculo puede afectar negativamente su diagnóstico y tratamiento. Una distancia aceptable entre terapeuta y paciente se requiere para una correcta intervención clínica por parte del terapeuta. Si el paciente tiene conocimiento de la vida privada del terapeuta, dicho conocimiento puede afectar el desarrollo psicológico autónomo del paciente o de su sistema de creencias. La relación terapéutica debe centrarse en resolver los problemas del paciente y no en las ideas o problemas del terapeuta. Las ideas o el sistema de creencias del terapeuta, sin son conocidas claramente por el paciente pueden actuar como una ideología opresora no permitiendo que el paciente piense por sí mismo, no ayudándolo, tal como diría Freire en su proceso de concientización acerca de su opresión. El paciente debe descubrir por sí mismo, a través del diálogo con su terapeuta, al igual que un educador dialoga con su educando, las condiciones de su opresión.

Finalmente, se puede afirmar que la relación terapeuta - paciente es similar a la relación educador - educando en el contexto de una educación o psicoterapia para la liberación. 
El antagonismo entre las dos concepciones, la "bancaria", que sirve a la dominación, y la problematizadora, que sirve a la liberación, surge precisamente ahí. Mientras la primera, necesariamente mantiene la contradicción educador - educandos, la segunda realiza la superación. (Freire, 1985. p.85).

En este apartado el educador y sus educandos deconstruyen el concepto de amistad y el concepto de relación terapéutica con el fin de establecer la diferencia entre ambos. Reflexionan acerca del concepto de distancia aceptable. Comprenden el porqué de la importancia de proteger el sistema de creencias del paciente. Reflexionan acerca del concepto de ideología opresora y del concepto de concientización. Analizan la diferencia entre la concepción bancaria de la educación y la concepción problematizadora.

\subsection{La relación terapéutica no es una relación amorosa}

El amor entre terapeuta y paciente no está aceptado debido a que afecta la cura del paciente. No obstante, existe desde el punto de vista psicoanalítico el concepto de amor de transferencia, es decir, el amor del paciente hacia el terapeuta que facilita al terapeuta el logro de la cura por parte del paciente. Este es un amor de carácter técnico para el terapeuta, pero puede no serlo para el paciente. Por ello, el terapeuta debe manejar esta situación con mucho cuidado.

Una situación similar puede ocurrir entre un educador y su educando. Aquí también se da un amor de transferencia que se sustenta en la admiración del alumno por el conocimiento de su maestro y que puede facilitarle al alumno su proceso de aprendizaje. No obstante, al igual que el terapeuta, el maestro debe indicarle al alumno que su relación es estrictamente educativa, especialmente si el alumno es menor de edad.

En este apartado el educador y sus educandos deconstruyen y analizan el concepto de amor de transferencia, uno de los conceptos más importantes aportados por la teoría psicoanalítica. Tratan de comprender cómo el amor de transferencia opera en la relación terapéutica como así también en la relación pedagógica. Nuevamente, parten de lo más cercano, de su propia experiencia subjetiva como educandos y muchos de ellos, como pacientes en su proceso de psicoterapia personal.

\subsection{La relación terapéutica no incluye relaciones sexuales}

Las relaciones sexuales entre el terapeuta y su paciente están prohibidas en casi todos los países del mundo. Debido a la naturaleza del amor de transferencia ya descripto, un hábil terapeuta puede usar este fenómeno psicológico con el fin de satisfacer sus necesidades sexuales no pudiendo en general el paciente defenderse de esta acción. Situaciones similares pueden producirse también en la relación educador 
- educando. Estas conductas por parte de terapeutas y educadores están consideradas en diversos códigos penales del mundo como abuso sexual.

En este apartado educador y educandos reflexionan acerca de la presencia de relaciones sexuales en la relación terapéutica y en la relación pedagógica como abuso sexual. Para ello, deconstruyen el concepto psicológico de abuso sexual. Algunos alumnos comparten sus propias experiencias personales.

\subsection{La relación terapéutica es siempre una relación de poder}

La relación terapéutica es siempre una relación de poder porque el terapeuta, como profesional de la salud mental tiene un conocimiento científico acerca del funcionamiento de la mente humana que su paciente no tiene. Este poder producto de su conocimiento técnico es impuesto sobre su paciente. Existen modelos de tratamiento psicoterapéutico que han enfatizado el poder del psicoterapeuta sobre el paciente como el psicoanálisis tradicional y la psiquiatría pasada o actual. Un ejemplo opuesto lo constituye la psicoterapia feminista que se ha ocupado de equilibrar la situación de poder diferencial entre terapeuta y paciente a través del uso de la estrategia psicológica denominada Self - Disclosure (del inglés) que en castellano significa Auto-Revelación. Dicha estrategia indica que el psicoterapeuta revela algo importante y personal de sí mismo siempre y cuando sea terapéuticamente útil para el logro de la cura del paciente. Esta estrategia ha sido muy fuertemente criticada por psicoterapeutas que usan la psicoterapia psicoanalítica tradicional donde pretenden reforzar su poder terapéutico sobre el paciente a través del uso del amor de transferencia con el fin de alcanzar la cura del paciente o satisfacer sus propias necesidades narcisísticas. Finalmente, la psiquiatría a lo largo de su historia también ha sostenido una postura que refuerza el poder del psiquiatra sobre sus pacientes. La postura de la psiquiatría actual se sostiene a través del llamado modelo médico ${ }^{3}$. Dicho modelo impone una ideología opresora sobre sus pacientes porque no tiene en cuenta el deseo de sus pacientes o su potencial capacidad de liberarse de la opresión de su enfermedad sin necesidad de recurrir al uso de psicofármacos. De igual manera, el educador tradicional es criticado por Freire porque usa su conocimiento con el fin de imponer una pedagogía de la opresión sobre sus alumnos. Este fenómeno fue descrito como educación bancaria que defiende el orden social dominante porque impide el proceso de concientización que sería inherente a una pedagogía de la liberación. Esto sucede porque:

3 El modelo médico es un modelo de tipo biologicista que ha tratado de encontrar marcadores biológicos para las enfermedades mentales sin mucho éxito hasta el momento. Dichos marcadores pueden ser los niveles de sangre de alguna sustancia, posibles alteraciones cromosómicas o genéticas. El modelo médico utiliza características biológicas en forma estadística, siendo muy discutible sus explicaciones causales. Este modelo es de tipo funcionalista y en general no considera los factores psicosociales, culturales o políticos en sus diagnósticos y tratamientos. 
En su actuación política, las élites dominantes son eficientes en el uso de la concepción "bancaria" (en la cual la conquista es uno de sus instrumentos) porque, en la medida que desarrollan una acción que estimula la pasividad, coincide con el estado de "inmersión" de la conciencia oprimida. (Freire, 1985, p. 110).

De igual forma la psiquiatría ha contribuido a naturalizar las relaciones sociales opresivas negando su influencia y patologizando a individuos como portadores de desórdenes mentales de naturaleza biogenética creando una psicoterapia de la opresión.

En este apartado educador y educandos deconstruyen el concepto de poder. Analizan la naturaleza del poder científico - técnico. Reflexionan acerca de la estrategia feminista de auto - revelación y la posición de sus críticos. Deconstruyen el concepto de modelo médico y su estrategia de poder. Reflexionan acerca de la pedagogía de opresión y de la psicoterapia de opresión.

\subsection{La relación terapéutica siempre incluye fenómenos transferenciales, contratransferenciales, de encuadre y de alianza terapéutica}

A comienzos del siglo XX Freud fue uno de los primeros investigadores que se dedicó a reflexionar acerca de la importancia de la relación entre el terapeuta y su paciente. Tal como indican Corbella y Botella (2009) para Freud esta relación presentaba tres áreas fundamentales: La primera la denominó transferencia y se refería a la identificación inconsciente del paciente con el terapeuta. La segunda la denominó contratransferencia y se refería a la conexión inconsciente del terapeuta con su paciente. La tercera área de interés para Freud (1913) la constituyó la conexión positiva del paciente con el tratamiento y la persona del psicoterapeuta. Esta tercera área fue denominada posteriormente alianza terapéutica. Este último concepto fue relacionado con el concepto de encuadre 4 en psicoanálisis.

El pensamiento psicoanalítico destacó la importancia del aspecto inconsciente de la relación terapéutica subestimando su parte consciente. Desde una postura opuesta, el pensamiento cognitivo destacó la importancia del aspecto consciente de la relación terapéutica subestimando su parte inconsciente. Ambas posturas recurren al denominado principio de simplificación desarrollado por Morin (1999). Mediante dicho principio simplifican la naturaleza de la relación terapéutica usando las operaciones de separación y disyunción.

En concordancia con lo señalado, educador y educandos partiendo del pensamiento de Morin intentarán resolver el problema planteado, o sea, tratarán de

4 Según Etchegogen (1986) el encuadre supone fijar como constantes las variables de tiempo y espacio estableciendo algunas normas que determinan los roles del paciente y del terapeuta en relación a la tarea terapéutica que se pretende efectuar. 
corregir el principio de simplificación empleado por la psicoterapia psicoanalítica y por la psicoterapia cognitiva. Esto se logra, tratando de integrar el saber proveniente de la psicoterapia psicoanalítica con el saber proveniente de la psicoterapia cognitiva, comprendiendo que la parte inconsciente y la parte consciente de la mente humana, constituyen una unidad, es decir, la mente humana está integrada por dos partes contradictorias ubicadas en el mismo nivel de realidad. Deberán considerar que la relación terapéutica, la relación entre terapeuta y paciente se logra a través de la articulación de la mente del terapeuta con la mente del paciente. Esta articulación de mentes se da a partir de la articulación de la parte consciente e inconsciente de la mente del terapeuta con la articulación de la parte consciente e inconsciente de la mente del paciente. Badilla Saxe (2008) nos dice:

Morin, por su parte, con su método de pensamiento complejo, nos recuerda que el aprendizaje consiste en "abrazar" el conocimiento como un todo, entendiendo las interrelaciones entre los conceptos. Mira la evolución del pensamiento como una espiral que va avanzando en "bucles" que regresan, eventualmente, a punto conocido, aunque mirando desde diferente perspectiva y si bien en educación estamos adoptando el discurso del pensamiento de Morin, con frecuencia hacemos todo lo contrario. Los planes de estudio están fragmentados, las materias separadas y delimitadas y con poca o ninguna relación entre ellas (como los ladrillos de una construcción, que aunque están uno al lado del otro, no se interrelacionan). (Badilla Saxe, 2008, p.1)

Teniendo en cuenta la reflexión de Badilla Saxe acerca del pensamiento de Morin, en este artículo sostenemos la importancia de "abrazar el conocimiento como un todo entendiendo las interrelaciones entre los conceptos". Siguiendo esta premisa, educador y educandos establecen las interrelaciones entre la mente del terapeuta y la mente del paciente en el contexto de la relación terapéutica. A su vez, interrelacionan los conceptos de consciente e inconsciente, como partes constituivas, contradictorias, ubicadas en un mismo nivel de realidad y que constituyen una unidad que denominamos mente humana.

\subsection{La relación terapéutica es un sistema adaptativo complejo}

El concepto de sistema fue usado por primera vez por el filósofo y matemático Leibniz en 1666 definiéndolo como "totalidad de elementos". Por ello, un sistema puede definirse como un conjunto de elementos que se encuentran en interacción. Esta interacción puede presentarse como un intercambio de energía, de materia, o de información. Cuando este intercambio se produce, emergen propiedades desconocidas a nivel de las partes componentes del sistema consideradas por separado.

En este artículo y en la asignatura Pre-Práctica se afirma que el sujeto humano es un sujeto complejo. Debido a su naturaleza compleja el sujeto humano también es un sistema, un sistema que podemos considerar como sistema complejo. Pozzoli 
(2006), en su texto sobre el sujeto de la complejidad nos dice que el "sujeto complejo es un sujeto organizado", es decir, organiza e influye el contexto del cual forma parte. El sujeto complejo obtiene energía, información y organización de la realidad externa, es decir, que el sujeto complejo presenta una auto-organización dependiente del medio, pero el sujeto complejo tal como lo indica nuevamente Pozzoli, M (2006) es "Unitas-Multiplex" significando que el sujeto complejo como perteneciente a la especie humana mantiene una relación compleja de naturaleza dialógica y recursiva entre la unidad y la diversidad y entre la diversidad en la unidad.

El educador y sus educandos construyen, reflexionan, vinculan el concepto de sistema con el concepto de sujeto complejo. A su vez, exploran como el concepto de sistema puede aplicarse al concepto de relación terapéutica. Partiendo de la comprensión de la naturaleza del sujeto complejo como "Unitas-Multiplex" pueden expandirlo y entender cómo el sujeto complejo construye relaciones complejas.

El sujeto complejo es un sistema como los descriptos en la teoría de los sistemas y como todo sistema está sujeto al principio de circularidad. El sujeto complejo como sistema está compuesto por subsistemas y a su vez, integra sistemas más amplios. El concepto de interacción, el concepto de sistema y el concepto de red constituyen un modo de organización que describe la naturaleza del sujeto complejo.

Si consideramos al sujeto humano como un sujeto complejo podemos concluir que las relaciones que el sujeto humano construye también son complejas y entre estas relaciones encontramos a la relación terapéutica. Por ello es importante que el educador y sus educandos puedan definir que entienden por sistema complejo, para luego adentrarse al concepto de relación terapéutica como sistema adaptativo complejo. Para ello, educador y educandos siguen la reflexión de Sánchez Ballesteros (2006) que define con claridad que son los sistemas complejos:

Los sistemas complejos son dinámicos y no lineales, turbulentos, no predecibles con exactitud; su funcionamiento no se basa en un algoritmo o patrón por encima de la conducta de sus elementos, sino en relaciones; esa interacción ocurre gracias a una entrada de información proveniente del entorno, provocando una salida o resultado conductual del sistema hacia el entorno.

La no linealidad del sistema significa la ruptura de la concepción de la necesidad de una causa con su efecto determinado: una perturbación o entrada de información al sistema, por mínima que sea, tiene efectos desproporcionados. Lo anterior se puede ejemplificar en el llamado efecto mariposa, que alude al sistema climático, en el que una perturbación como el aleteo de una mariposa en el continente americano puede provocar un ciclón en el continente asiático...Otro ejemplo, pero ahora en el ámbito cultural, lo encontramos en la actividad epistémica del individuo en el acto de la interpretación, donde sopesa diversas variables como sus experiencias, prejuicios y el acontecimiento del ser en un 
lenguaje con multitud de sentidos, obteniendo una interpretación de lo que le acontece, interpretación que puede cambiar de un tiempo a otro sin una causalidad proporcional; es decir, que a determinados factores epistémicos necesariamente resulta una, y sólo una concepción específica del acontecimiento (Sánchez Ballesteros, 2006, p. 1).

A partir de lo indicado por Sanchez Ballesteros (2006), los educandos describen la naturaleza de los sistemas complejos como dinámicos y no lineales, turbulentos, no predecibles con exactitud. Educador y educandos parten de la idea de que el terapeuta y su paciente constituyen un sistema complejo porque el terapeuta y el paciente presentan una conducta (entendida como respuesta a un estímulo) que puede describirse como dinámica (en movimiento) porque no es fija, no es mecánica, propia de un sistema complejo. La dirección del sistema complejo, su conducta, no presenta un carácter lineal. No se dirige de un punto a otro cubriendo un trayecto que podría representarse gráficamente como una línea recta. La conducta del sistema terapeuta - paciente es turbulenta, es decir, está sujeta a la acción del medio ambiente externo e interno. El medio ambiente externo lo constituyen los estímulos provenientes del mundo exterior que son procesados por la mente consciente del sistema complejo. El medio ambiente interno lo constituyen estímulos provenientes del mundo interior siendo procesados por la mente inconsciente en situación transferencial afectando lógicamente a los dos componentes del sistema complejo (terapeuta - paciente). En lo inconsciente se encuentran las causas originarias de las psicopatologías procesadas por los dos componentes del sistema complejo. No obstante, una parte del mismo sistema complejo (terapeuta) va a ayudar a la otra parte (paciente) a concretar el proceso de la cura $^{5}$ entendida como el logro de la verdad subjetiva del paciente que se encuentra determinada por su posición subjetiva en una de las tres estructuras clínicas.

Educador y educandos, siguiendo a Sanchez Ballesteros (2006) analizan el funcionamiento del sistema complejo terapeuta - paciente explorando la interacción que ocurre gracias a una entrada de información proveniente del mundo externo o del mundo interno y cómo se provoca una salida o resultado conductual del sistema hacia ambos.

A su vez, la no linealidad del sistema "significa la ruptura de la concepción de la necesidad de una causa con su efecto" (Sanchez Ballesteros, 2006, p.1), idea no sustentada por la lógica clásica; pero sí sustentada por la lógica de la complejidad. También se afirma que "una perturbación o entrada de información al sistema, por mínima que sea, tiene efectos desproporcionados" (Sanchez Ballesteros, 2006, p.1). En la relación terapéutica, la no linealidad del sistema, significa que

5 Siguiendo la reflección de Dylan Evans (2015) en su Diccionario Introductorio de Psicoanálisis Lacaniano, el concepto de "cura" se utiliza en este artículo en un sentido completamente distinto al seguido por la medicina. La meta de la cura para Lacan no es sanar o curar en el sentido de producir una psique perfectamente sana. Las estructuras clínicas (neurosis, psicosis y perversión) son consideradas esencialmente incurables y el tratamiento psicoanalítico se propone únicamente llevar al analizante a expresar su verdad subjetiva. 
no existe una causa y su efecto; pueden existir diversas causas y diversos efectos de naturaleza consciente o inconsciente, que lógicamente pueden producir efectos desproporcionados a nivel del sistema total incluyendo su propia destrucción, es decir, destruyendo la relación terapéutica.

Educador y educandos definen y comparan la naturaleza de la lógica clásica en relación a la lógica de la complejidad considerando la no linealidad de todo sistema complejo donde se incluye a la relación terapeuta - paciente. Luego reflexionan acerca del concepto de evolución y de cambio en el contexto de la relación terapéutica.

Al ser un sistema adaptativo complejo, la relación terapéutica requiere para el mantenimiento de las partes del sistema conectadas, un constante flujo de energía y un constante flujo de información. Cuando el medio cambia y el sistema se adapta podemos hablar de evolución. A través de este proceso evolutivo que llamaremos también adaptativo, el sistema modifica su medio. La evolución que mencionamos aquí se concibe como un proceso incremental de orden, complejidad y verdad en el sentido lacaniano ya explicado.

Educador y educandos reflexionan acerca de la perspectiva dinámica de la relación terapéutica y de cómo se crean sistemas de mayor complejidad. Es decir, desde una perspectiva dinámica se puede concebir la relación terapéutica como un sistema adaptativo complejo. Esto es así porque el terapeuta y el paciente como sujetos complejos crean un nuevo sistema de mayor complejidad en permanente proceso coevolutivo a través de la interacción entre ambos. Palombo nos dice al respecto:

This is the dyad composed of the patient and the analyst; and both patient and analyst are systems. These are systems within systems. We want to know how changes in the ecosystem formed by the patient and the analyst can increase the fitness of the patient. My hipothesis is that adaptive change in the patient results from the coevolution of the therapeutic dyad in the analytic ecosystem. A better adapted ego emerges in the patient as the patient and the analyst become better adapted to one another ${ }^{6}$ (Palombo, 1999, pp. 1-2)

El educador y sus educandos construyen el concepto de comportamiento emergente y de cómo se originan las diversas psicopatologías; porque en el sistema adaptativo complejo el comportamiento emerge de la interacción de sus elementos; es decir, de la interacción del paciente y su terapeuta, lógicamente, cada uno de ellos por separado, es un sistema adaptivo complejo menos evolucionado. Esto es así, porque cada uno está en interacción con su mundo externo e interno; cada uno de ellos tiene la

6 Esta es la diada compuesta por el paciente y el analista; el paciente y el analista, ambos son sistemas. Son sistemas dentro de sistemas. Lo que queremos saber es cómo los cambios producidos en el ecosistema formado por el paciente y el analista pueden incrementar la salud y adaptabilidad del paciente. Mi hipótesis es que los cambios adaptativos en el paciente son resultado de la coevolución de la diada terapéutica en el ecosistema psicoanalítico. Un yo más saludable, más adaptado, emerge en el paciente cuando el analista y el paciente logran mejor adaptarse el uno al otro. 
capacidad de movilizar la parte consciente e inconsciente de su mente para lograr una mejor adaptación a sus mundos; cada uno de ellos debe enfrentarse eventualmente a los contenidos traumáticos, origen de sus propias psicopatologías que son removidos de la consciencia y depositados en lo inconsciente para proteger la integridad y solidez del $\mathrm{yo}^{7}$, con la finalidad por parte de este último, de poder enfrentarse más exitosamente con su mundo interior y exterior. De todas maneras, estos contenidos traumáticos no son eliminados totalmente de la mente sino que continúan afectando al yo desde lo inconsciente siendo origen de diversas psicopatologías. Por ello, a través de la relación de los componentes del sistema complejo terapeuta - paciente dichas psicopatologías emergen durante el proceso terapéutico. Estas psicopatologías constituyen dificultades emocionales, que el paciente no puede vencer por sí mismo y se da cuenta que necesita ayuda externa para enfrentarlas. Entonces, el terapeuta lo ayuda a entenderlas, a examinar su funcionamiento con el fin de enfrentarlas, indicándole cómo acceder a su saber inconsciente donde ellas se encuentran. Por ello se puede afirmar que el sistema complejo terapeuta - paciente es un sistema adaptativo complejo más evolucionado porque puede integrar el saber inconsciente y el saber consciente como producto de la relación terapéutica en transferencia produciendo en el paciente un comportamiento emergente de carácter más evolucionado, es decir, más verdadero en sentido lacaniano y menos psicopatológico, representando una mejor adaptación del paciente a su mundo interno y externo.

A su vez, educador y educandos comparan y reflexionan acerca de los conceptos de salud y enfermedad desde la perspectiva de una relación terapéutica como sistema adaptativo complejo concluyendo que un óptimo nivel del funcionamiento del sistema se logra cuando terapeuta y paciente se adaptan entre sí de la mejor manera posible. Esto debe alcanzarse a nivel transferencial y a nivel de alianza terapéutica. Cuando las interacciones de los elementos del sistema adaptativo complejo están rigidizadas, como indica Caparrós (2009) es cuando se produce la enfermedad (la no verdad subjetiva lacaniana) y cuando la relación terapéutica no está rigidizada (se debilita la enfermedad) es cuando el terapeuta y el paciente trabajan conjuntamente con el fin de promover la emergencia de un yo más saludable en el sentido de verdadero, mejor adaptado, del paciente a su medio.

Educador y educandos analizan y comparan los conceptos de transferencia, contratransferencia y alianza terapéutica. Deducen que dichos conceptos representan posturas teóricas en oposición. Desde una posición dinámica se ha destacado la importancia de los fenómenos transferenciales inconscientes sobre lo consciente. Por otra parte, desde una postura cognitiva se resalta la importancia de lo consciente sobre los fenómenos transferenciales. Estas posturas teóricas se basan en el uso del paradigma de la simplificación que incluye la aplicación de las operaciones de separación y disyunción. Separanloque debiera estarunido: Separanloconscientedelainconsciente.

7 En su diccionario de psicoanálisis Laplanche y Pontalis (1967) indican que el "yo" es una instancia que Freud distingue del ello y del superyó en su segunda teoría del aparato psíquico. 
Se puede afirmar que la explicación anterior si bien es correcta, no es completa. Para alcanzar una explicación más completa debemos recurrir a dos conceptos centrales del pensamiento complejo: El concepto de niveles de realidad y el concepto del tercero incluido. Ambos conceptos deberán ser compredidos por los educandos con el fin de entender la naturaleza de la reducción puesta en práctica por el psicoanálisis y la teoría cognitiva. Para ello, educador y educandos parten de un análisis como el efectuado por Max Neef (2004) acerca de los niveles de realidad. Dicho autor afirma que la realidad presenta diversos niveles. También que es posible pasar de un nivel a otro; pero ésto sólo se logra mediante una ruptura que es producto de este pasaje. Un ejemplo de esto lo constituye el pasaje de contenidos psíquicos de lo consciente a lo inconsciente; o el pasaje de contenidos psíquicos de lo inconsciente a lo consciente. Lo consciente es un nivel de realidad que está regido por leyes y conceptos propios. De la misma manera, lo inconsciente es otro nivel de realidad que también está regido por leyes y conceptos propios.

A su vez, Max-Neef (2004) señala que la lógica del tercero incluido es una lógica de la complejidad porque tiene por objeto la incorporación del tercero excluido. El tercero excluido es un concepto propio de la lógica clásica. El psicoanálisis y la teoría cognitiva basan sus ideas en el uso de la lógica clásica teniendo dificultades para notar que la diferencia entre lo inconsciente (transferencia - contratransferencia) puede coexistir y de hecho coexiste con su contradictorio, lo consciente. Es decir, lo inconsciente y lo consciente son parte del mismo nivel de realidad.

En la asignatura Pre-Práctica el educador y sus educandos concluyen que la relación terapéutica está constituida por el terapeuta y el paciente como sujetos complejos que a su vez interaccionan en situación transferencial y de alianza terapéutica, creando un sistema adaptativo más complejo: y facilitando la emergencia de un yo del paciente más adaptado, más verdadero, en el sentido lacaniano del término. A su vez, destaca, mediante la lógica del tercero incluido, que la relación terapéutica abarca también lo consciente y lo inconsciente que constituyen un mismo nivel de realidad integrado por dos elementos contradictorios.

\section{CONGLUSIÓN}

En este artículo se ha explicado la importancia del aprendizaje del concepto de relación terapéutica por futuros psicoterapeutas, es decir, de la relación entre el terapeuta y el paciente en el proceso de psicoterapia y en los cambios que el paciente pueda realizar, con el fin de mejorar su salud mental. Por ello, se ha hecho saber que la relación terapéutica es el contenido central del curso Pre-Práctica que los estudiantes deben aprobar antes de iniciar su práctica de residencia como psicoterapeutas en la carrera de maestría en espiritualidad y psicoterapia de la universidad Wilfrid Laurier de Canadá. Esto es así, porque la calidad de la relación terapéutica, es decir, la calidad de la relación entre el terapeuta y el paciente puede afectar el tratamiento 
y su resultado. Si la calidad de la relación terapéutica es positiva, el tratamiento y su resultado también lo serán. Durante cualquier tratamiento se espera que el paciente pueda mejorar su autoestima, su capacidad en la toma de decisiones y que pueda reducir la influencia negativa de su enfermedad.

En este trabajo se han analizado las similitudes y las diferencias entre la relación terapéutica (terapeuta - paciente) y la relación pedagógica (educador - educando). Se ha explicado cómo el educador y los educandos construyen el concepto de relación terapéutica siguiendo el pensamiento de Piaget. A su vez, se ha analizado el uso del poder por parte del psicoterapeuta con el propósito de ayudar al paciente a liberarse de la opresión ejercida por la enfermedad mental. También se han descripto otras formas de opresión de carácter social. Para ello se introdujo el concepto de interseccionalidad. El uso del poder fue abordado en la relación terapéutica y en la relación pedagógica siguiendo la perspectiva del pensamiento de Freire.

Teniendo en cuenta las reflexiones de Freire, se describió como a través del uso del poder en la relación pedagógica se puede deconstruir el uso del poder en la relación terapéutica. Esto es así porque en la relación pedagógica, el educador tradicional sigue el modelo bancario de educación donde deposita su conocimiento en la supuesta mente en blanco de sus educandos. A través de esta acción, impone su percepción de la realidad creada por las clases dominantes. Esta pedagogía de la opresión no permite que los educandos piensen por sí mismos, afirmando su autoestima, su capacidad de toma de decisiones, o desarrollando un pensamiento crítico, o sea, impide la concientización acerca de la posición de los enducandos en relación a su contexto social opresivo. De la misma manera, la relación terapéutica puede repetir el modelo opresor descripto por Freire (1968) cuando dicha relación no le permite al paciente afirmar su autoestima, su capacidad de toma de decisiones o el desarrollo de un pensamiento crítico. El enfermo mental se encuentra entonces, al igual que el educando descripto por Freire, frente a una psicoterapia bancaria, que es el tipo de psicoterapia que se sostiene a través del uso de psicofármacos o mediante el reforzamiento del poder del psicoterapeuta, limitando la concientización y el pensamiento crítico del paciente en relación al abordaje de su enfermedad.

En la enseñanza de la relación terapéutica desde una perspectiva tradicional siguiendo el modelo bancario de educación propuesto por Freire se afirma el principio de simplificación desarrollado por Morin (1999) que es sostenido por la psicoterapia psicoanalítica o por la psicoterapia cognitiva. Debido a ello, el psicoterapeuta tradicional como el educador tradicional, no reflexiona acerca de un concepto transdisciplinario de relación terapéutica como producto de la integración de dos saberes en oposición provenientes de la psicoterapia psicoanalítica y de la psicoterapia cognitiva. No considera, que la mente del terapeuta y la mente del paciente, incluye lo inconsciente y lo consciente. Tampoco, toma en cuenta que la mente humana está integrada por dos partes contradictorias, en oposición, coexistiendo dialécticamente en un mismo nivel de realidad. Por ello, el pensamiento de Morin es de suma importancia porque permite la corrección del principio de simplificación. 
También en este artículo se describe como los educandos puede hacer un aprendizaje de los aspectos fundamentales de la relación terapéutica diferenciándola de las restantes relaciones humanas. Se establece que la relación terapéutica no puede considerase relación de amistad, ni relación amorosa y no incluyen en sí, la posibilidad de relaciones sexuales.

Asimismo, se ha descripto el proceso de aprendizaje del concepto de relación terapéutica como sistema adaptativo complejo. El educador y sus educandos parten de la naturaleza del sujeto humano como sujeto adaptativo complejo, es decir, un sujeto que tiene la capacidad de auto-organizarse en relación a su contexto. A su vez, consideran que paciente y terapeuta en la relación terapéutica, constituyen ambos, un nuevo y más evolucionado sistema adaptativo complejo. Debido a ello, terapeuta y paciente, deben adaptarse entre sí con el propósito de llegar a un óptimo nivel de funcionamiento. Esto debe lograrse a nivel transferencial y a nivel de alianza terapéutica. Siguiendo esta conceptualización educador y educandos concluyen que la enfermedad mental es un producto de la rigidización de los elementos del sistema adaptativo complejo y cuando la rigidización está reducida o no sucede; debido a una positiva situación transferencial y de alianza terapéutica, es cuando terapeuta y paciente trabajan conjuntamente con el fin de promover la emergencia de un yo más saludable, mejor adaptado, del paciente a su contexto.

Finalmente, se puede destacar que la tesis de este trabajo fue establecer la naturaleza de la relación terapéutica, su definición, con el fin de implementar un proceso de enseñanza - aprendizaje adecuado de la misma. Por ello se ha definido a la relación terapéutica como una relación intersubjetiva, de carácter profesional y de poder; conteniendo fenómenos transferenciales, contratransferenciales, de encuadre, de alianza terapéutica y fue descripta como sistema adaptativo complejo.

\section{BIBLIOGRAFÍA}

Acuña Bermúdez, Edgar Alfonso (2009). La Relación Psicoterapéutica: Reflexiones sobre Transferencia y Alianza Terapéutica, Secreto profesional y Confidencialidad. Revista de Psicoanálisis, Psicoterapia y Salud Mental, vol 2, n. 6.

Ávila Espada, Alejandro (2009). La psicoterapia psicoanalítica relacional: Conceptos fundamentales y perspectivas. Décimo Congreso Virtual de Psiquiatría. Interpsiquis. Psiquiatria.com

Badilla Saxe, Eleonora (2008). Dos ejemplos: Piaget y Morin. www.nacion.com/.../ ejemplos-Piaget-Morin_0_1021897826.html

Bakirer, Fabián (2005). Pensar el cambio social: las alternativas entre la desilusión ante el caos y la ilusión determinista. Revista: Comunidad de Pensamiento Complejo info@pensamientocomplejo.com.ar

Caparrós, Nicolás (2009). El psicoanálisis desde el paradigma de la complejidad. Átopos. Disponible en: http://atopos.exlibrisediciones.com/pdf_09/art3_0410.pdf 
Corbella, Sergi \& Botella, Luis (2003). La alianza terapéutica: historia, investigación y evaluación. Anales de psicología, vol 19, n. 2 (diciembre), pp. 205-221.

Evans, Dylan (1996). Diccionario Introductorio de Psicoanálisis Lacaniano. Buenos Aires: Paidos.

Freire, Pablo (1985). Pedagogía del oprimido. Buenos Aires: Siglo XXI Editores.

Gómez, Beatriz (2010). La relación terapéutica en Terapia Cognitiva. www.psiquiatria. com/psicoterapias.../la-relacion-terapeutica-en-tera...

Juárez, José Manuel y Canboni Salinas, Sonia (2012). Epistemología del pensamiento complejo. Reencuentro: Sujeto, subjetividady Educación Superior, n. 65, pp. 38-51.

Laplanche, Jean y Pontalis, Jean Bertrand (2001). Diccionario de Psicoanálisis. Buenos Aires: Paidos.

Martínez Gómez, Germán Iván (2015). La Filosofía de la Educación de Paulo Freire. Revista Internacional de Educación para la Justicia Social (RIEJS), 4(1), 55-70. doi: $10.15336 /$ riejs

Max-Neef, Manfred (2004). Fundamentos de la Transdisciplina. Valdivia: Universidad Austral de Chile. Disponible en ecosad.org

Moriello, Sergio Alejandro (2013). Investigación: Sistemas Complejos. Red Científica. http://www.redcientifica.com

Morin, Edgar (1999). Los siete saberes necesarios para la educación del futuro. París: Organización de las Naciones Unidas para la Educación, la Ciencia y la Cultura.

Palombo, Stanley (1999). The Emergent Ego: Complexity and Coevolution in the psychoanalytic Process. Connecticut: International Universities Press, INC

Pozzoli, María Teresa (2006). El sujeto de la complejidad. La construcción de un Modelo Teórico Transdisciplinar (eco-psico-socio-histórico-educativo). Polis: Revista Latinoamericana, n. 15, art. 9. ISSN 0717-6554, ISSN-e 0718-6568

Sánchez Nájera, Susana Aracele y Brahim, El Filali y López Ramirez, Victor Manuel (2013). El proceso de aprendizaje a través del pensamiento complejo. Eleventh LACCEI Latin American and Caribbean Conference for Engineering and Technology (LACCEI'2013) "Innovation in Engineering, Technology and Education for Competitiveness and Prosperity" August 14 - 16, 2013 Cancun, Mexico

Sánchez Ballesteros, Enrique (2006) ¿Qué es el fenómeno de lo complejo? La Ciencia y el hombre: Revista de Divulgación Científica y Tecnología de la Universidad Veracruzana, vol. XIX, n. 3, art. 5.

Santibáñez, Patricia. (2003). La alianza terapéutica en Psicoterapia: El "inventario de Alianza de Trabajo" en Chile. PSYKHE, vol. 12, n. 1, pp. 109-118.

Tobón, Sergio \& Fernández, José (2004). La terapia desde una perspectiva compleja. Quinto Congreso Virtual de Psiquiatría. Interpsiquis. Psiquiatria.com. 